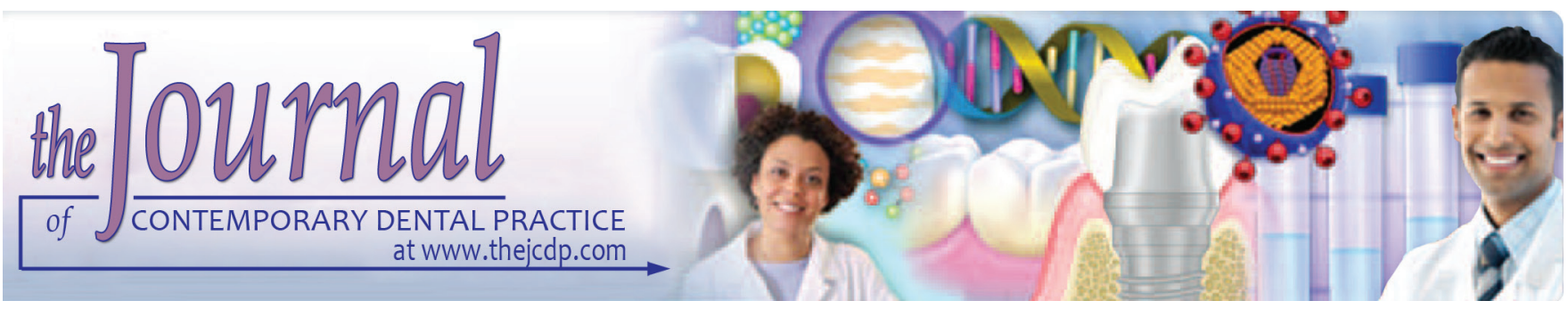

\title{
Catalogue of Interactive Learning Objectives to improve an Integrated Medical and Dental Curriculum
}

\author{
${ }^{1}$ Benjamin Mahmoodi, ${ }^{2} \mathrm{~K}$ Sagheb, ${ }^{3} \mathrm{Ka}$ Sagheb, ${ }^{4} \mathrm{P}$ Schulz, ${ }^{5} \mathrm{~B}$ Willershausen, ${ }^{6} \mathrm{~B}$ Al-Nawas, ${ }^{7} \mathrm{C}$ Walter
}

\begin{abstract}
Introduction: Online learning media are increasingly being incorporated into medical and dental education. However, the coordination between obligatory and facultative teaching domains still remains unsatisfying. The Catalogue of Interactive Learning Objectives of the University Clinic of Mainz (ILKUM), aims to offer knowledge transfer for students while being mindful of their individual qualifications. Its hierarchical structure is designed according to the Association for Dental Education in Europe (ADEE) levels of competence.
\end{abstract}

Materials and methods: The ILKUM was designed to establish a stronger interconnection between already existing and prospective learning strategies. All contents are linked to the current lectures as well as to e-learning modules, e.g., clinical case studies and OR videos. Students can conduct self-examinations regarding specific learning objectives. Since 2007, ILKUM has been developed and analyzed regarding its acceptance among dental students.

Results: These improved e-learning techniques foster time and location-independent access to study materials and allow an estimation of the knowledge achieved by students. Surveys of our students clearly show a large demand for upgrading ILKUM content $(89 \% ; n=172)$ with integrated self-testing $(89 \% ; n=174)$. In parallel to the advancement of our e-learning offering, a portion of internet-based learning is constantly rising among students.

Conclusion: The broad acceptance and demand for the development of ILKUM show its potential. Moreover, ILKUM grants fast, topic-oriented querying of learning content without time and locale limitations as well as direct determination of the individually needed knowledge conditions.

\footnotetext{
${ }^{1,5}$ Department of Operative Dentistry and Periodontology University Medical Center of the Johannes Gutenberg University Mainz, Germany

2-4,6,7Department of Maxillofacial Surgery, University Medical Center of the Johannes Gutenberg University, Mainz, Germany

Corresponding Author: Benjamin Mahmoodi, Department of Operative Dentistry and Periodontology, University Medical Center of the Johannes Gutenberg University, Mainz, Germany e-mail: benjamin.mahmoodi@unimedizin-mainz.de
}

Clinical significance: The long-term goal of the ILKUM project is to be a sustainable, important additional modality of teaching and training for dental and medical students.

Keywords: Computer-assisted learning, e-Learning, Interactive learning, Level of competence, Medical and dental curriculum.

How to cite this article: Mahmoodi B, Sagheb K, Sagheb K, Schulz P, Willershausen B, Al-Nawas B, Walter C. Catalogue of Interactive Learning Objectives to improve an Integrated Medical and Dental Curriculum. J Contemp Dent Pract 2016;17(12):965-968.

\section{Source of support: Nil}

Conflict of interest: None

\section{INTRODUCTION}

In the past, education of dental school graduates largely occurred by means of textbooks, handouts, and notes taken during courses. In recent years, students have been increasingly exposed to e-learning techniques. Onlinebased learning is predicted to become a successful, revolutionary method of education. It has been shown that a significant percentage of higher educational institutes believe that online learning is crucial to their long-term strategy. ${ }^{1-4}$ However, many of the present methods not only fail to satisfactorily combine the facultative and obligatory tutoring options, they also fail to meet the professional performance requirements. ${ }^{5}$ Due to these shortcomings, the current e-learning methods are still not considered to be the primary medium for higher education. ${ }^{6}$ e-Learning media provides an impressive number of teaching options, but as a general precondition for them to be optimally effective, they all must be integrated into already existing training concepts.

This notion is supported by the Association for Dental Education in Europe (ADEE), which developed a draft called "Profile and Competences for the European Dentist - Update 2009." ${ }^{7}$ The guidelines subdivide the 
teaching schedule for dental education into three ascending competence levels:

- Level I: "Be familiar with": The student must be aware of basic theoretical knowledge, but no practical skills are required.

- Level II: "Have knowledge of": In addition to sound theoretical knowledge, basic practical experience as well as basic practical capabilities are required.

- Level III: "Be competent at": The student must possess strong theoretical and practical knowledge.

The acceptance of a newly offered teaching technique also requires a convincing balance between the teaching content offered by lecturers and the performance needs of the students. Our aim was to establish an interactive, multimedia learning catalogue named ILKUM, which would optimally combine course content and performance requirements. The teaching content is represented systematically in order to make navigation easy within the spectrum of learning objectives. Moreover, each learning unit is assigned to a semester-based proficiency level, contributing to the student's self-directed learning process. In a second step, the acceptance of ILKUM among the students was assessed.

\section{MATERIALS AND METHODS}

\section{Implementation of ILKUM}

The current teaching concept of our department can be described as follows. General topics of oral and maxillofacial surgery are taught in a problem-based and clinicalcase-oriented manner during the main lecture. Overall, emphasis is put on small student groups, which receive enhanced instructions during practical surgery courses that include training in essential technical skills. These lectures are electronically recorded and made available as problem-orientated learning intranet lectures. This provides time and location-independent access to the curriculum for the students. Interplay of the four essentials:

1. case-oriented clinical courses,

2. e-learning modules,

3. small student groups, and

4. e-examination/lecturer evaluation

creates the best possible learning and teaching environment. This approach introduces an interdisciplinary concept, promoting quality assurance particularly with respect to practical aspects during clinical education.

An interactive e-catalogue of learning objectives for the medical discipline "Oral and Maxillofacial Surgery" was established. The e-catalogue is a rigorous presentation of learning objectives according to the predefined catalogue specifications and is produced with consideration of the individual level of training. The levels of competence follow the standards set by the ADEE, and the underlying learning objectives are structured in modules within clinical topics.

The interactive content of the learning objectives catalogue is linked to the corresponding lectures and electronic learning modules. For all topics, the relevant literature (original articles, reviews, case reports) is added to provide a deeper insight into the specific subjects of clinical interest.

Due to the ever-changing nature of medical knowledge, continuous updating of the databases is conducted in close cooperation with both students and lecturers to constantly improve user friendliness as well as up-to-date medical knowledge. Furthermore, the modular design of the learning objectives catalogue facilitates adaptations and extensions at any time. An important direction for future developments is to expand the databases to comply with integrated and interactive examinations.

Security and privacy of personal data is ensured by means of individualized, password-protected access (encrypted connection) on the part of all users (lecturers, students, and moderators). No ethical was therefore needed.

\section{Evaluation of ILKUM}

To evaluate the impact of our e-learning concept on the students, we analyzed the acceptance of this kind of learning support among them. The acceptance and learning strategies of all dental students since 2008 were evaluated with an additional data sheet at the end of the semester's final exams. As well, at the end of the term, all dental students $(n=175)$ in the clinical part were questioned from 2008 up to 2010.

\section{RESULTS}

The following objectives were achieved by this educational project:

1. Coordination of dental curricula and the expected performance of students:

The discrepancy between course content and the performance requirements was minimized. For the first time, an understandable, accessible catalogue for all students was defined for oral and maxillofacial surgery.

2. Link between theoretical and practical aspects:

Strategies for theoretical teaching include differential diagnostic guidelines, whereas strategies for practical teaching include diagnosis-oriented symptomatology. The ILKUM catalogue is believed to ultimately achieve a more successful integration of these two teaching strategies. The clinical competence of the students is increased by the problem-oriented presentation of our comprehensive clinical case collection.

3. Interdisciplinary and interconnection of teaching:

The catalogue of learning objectives offers the possibility to directly create an interdisciplinary networking facility with neighboring disciplines. The close association with 
the proposals of the ADEE allows increased mobility of students within the European Union for a medium-term time period. It also enables the adaptation of educational developments in line with the Bologna process ${ }^{8}$ and in association with the European Credit Transfer System. ${ }^{9,10}$ 4. Improvement of teaching:

Students will develop individual teaching modules, working in small groups with the evidence-based medicine seminars in dentistry. The use of mobile devices (e.g., iPads) enhances the focus on the principle of "Blended Learning" within the context of small group teaching.

5. Quality management of teaching:

Our interactive catalogue has direct links to already existing topics of course content and e-learning modules. Through its modular architecture, it is possible to introduce new teaching and learning media in dental and medical education. Through the expansion of interactive selfmonitoring and continuous evaluation of knowledge by e-examinations at the end of each clinical semester, a high degree of quality assurance is achieved. This prospect is always complemented by close cooperation with students.

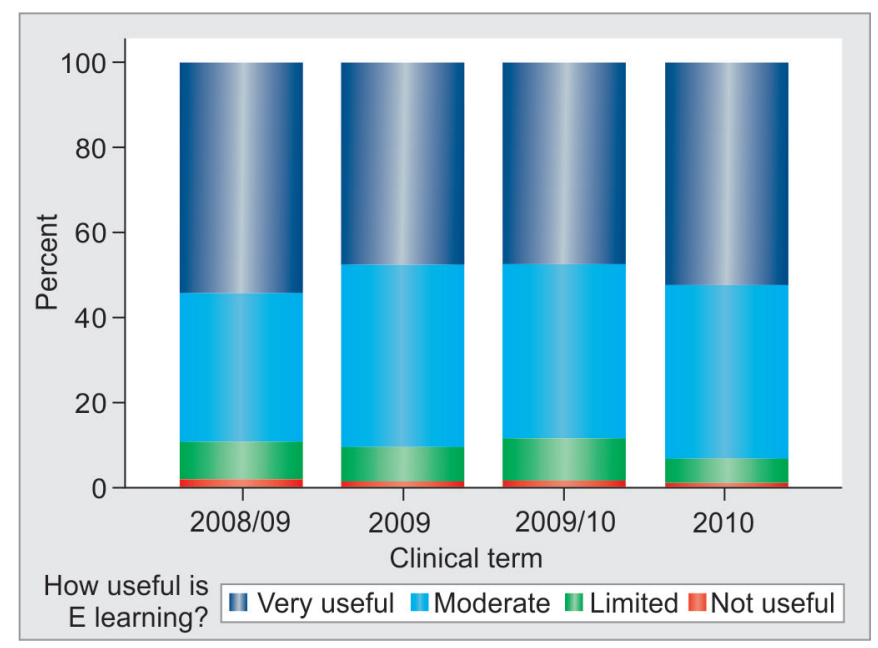

Graph 1: Students' acceptance of e-learning

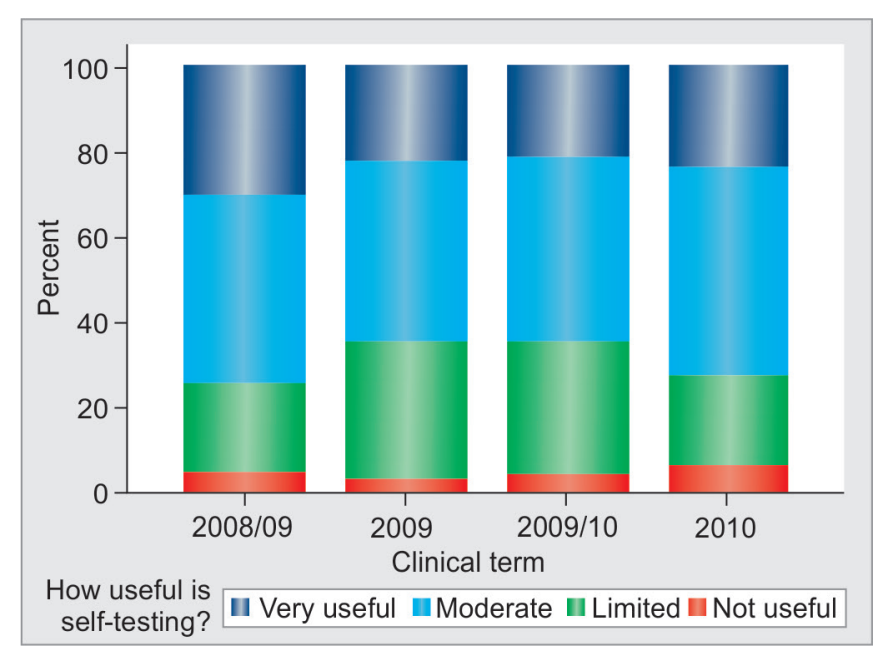

Graph 3: Students' acceptance of e-self-testing
6. Acceptance and learning strategies of the dental students at our clinic:

Approximately $50 \%$ of all students think e-learning is extremely reasonable; $40 \%$ like it, 9\% have doubts, and $1 \%$ are not interested (Graph 1 ). Around $90 \%$ of all students would like the internet-based e-learning to be constantly upgraded (Graph 2) and are highly interested in "e-selftesting" (Graph 3). The percentage of students using "internet-based learning" has been rising constantly (Graph 4).

\section{DISCUSSION}

The ILKUM catalogue enables students to inform themselves on the required level of competence and serves as a guiding tool throughout their education. Beyond case studies, ILKUM also allows access to specific course content and provides interactive self-evaluation exercises to assess knowledge levels. The ability to self-assess one's own competence is a crucial skill for all health care professionals. $^{11,12}$

By utilizing mobile devices, such as tablet computers and smartphones, we intend to reinforce the learning

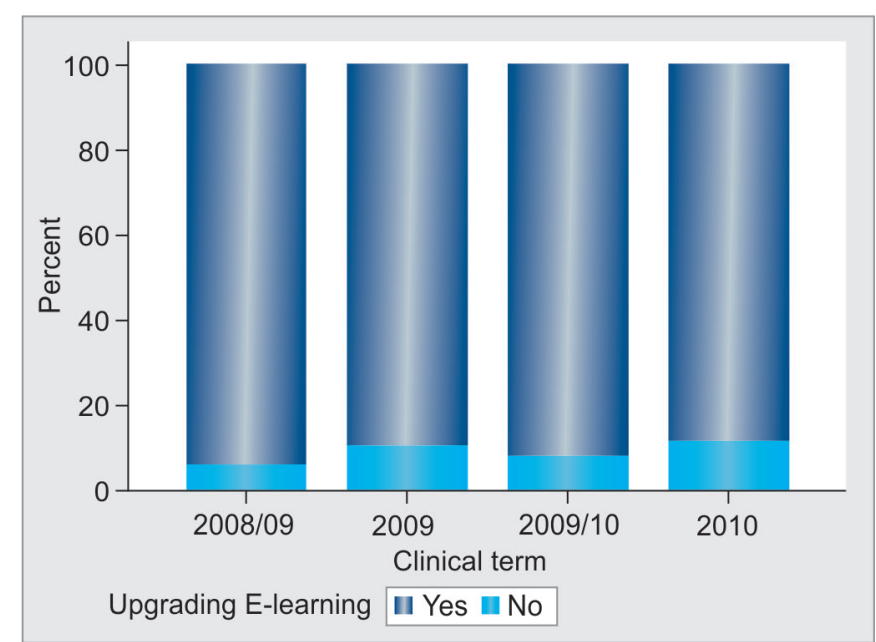

Graph 2: Students' demand for upgrading e-learning

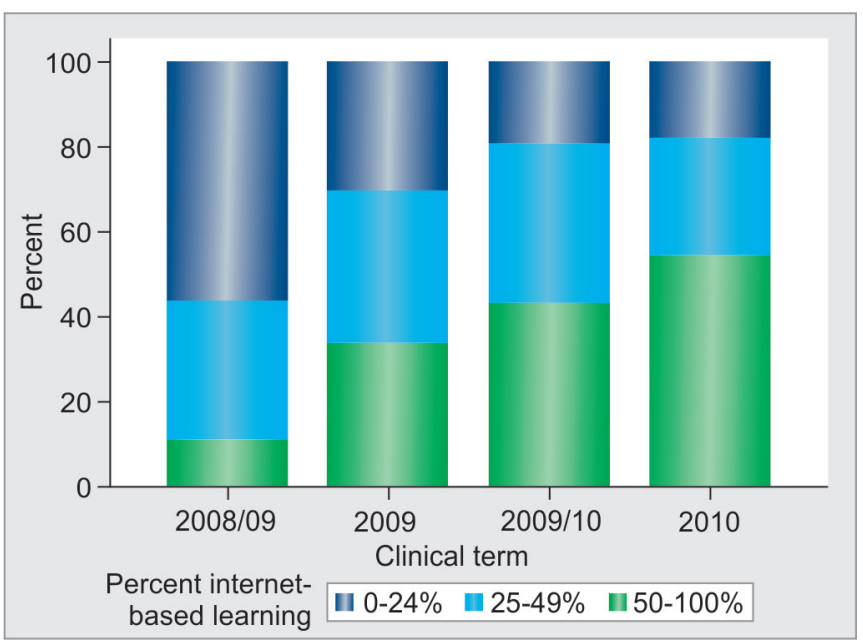

Graph 4: Percentage of students' internet-based learning 
impact of small student groups in the strict sense of blended learning. It has been well described that blended team-based learning positively influences students' attitudes toward learning. ${ }^{13,14}$ Moreover, the use of tablet computers is believed to improve Objective Structured Clinical Examination (OSCE). ${ }^{15,16}$

Since its introduction, the ILKUM platform has led to an increased acceptance of internet-based e-learning by students in dental medicine. Currently, various departments of the University Medical Center, Mainz, Germany use the ILKUM concept and rely on the ILKUM architecture.

The structuring of the e-learning platform in terms of learning objectives has promoted didactic discussions regarding the development of concepts in distinct teaching fields. The University Medical Center, Mainz, Germany participated in an ongoing nationwide debate regarding a national catalogue of learning objectives, and as a result, specific topics raised during this discussion were incorporated into ILKUM.

\section{CONCLUSION}

e-Learning has been established as an essential tool to improve dental and medical education within the last few years. But information overload as well as the way how computer-assisted learning can be better provided for students is under continuous discussion. Based on the concept of ADEE, we have developed an interactive e-learning database focusing on the Dental Curriculum (DC, Lernzielkatalog). In addition to the classical interactive construction, a modular level has been implemented to improve the reconciliation between the DC and the dental performance requirements.

The long-term goal of the ILKUM project is to be a sustainable, important additional modality of teaching and training for dental and medical students. Finally, the realization of this proposition should be achieved by embedding the catalogue in the "Regulations of Approbation" for physicians and for dentists. In addition, the prospective postgraduate education, e.g., training for residency, should and probably will rely on the ILKUM concept.

\section{AUTHORS' CONTRIBUTION}

KS carried out the study, was involved in the design and analysis, and contributed to the writing up of the paper. KJT and CW were involved in the statistical analysis and the write up of the paper. BM was involved in the design and contributed to the write up the paper. KaS was involved in the design and statistical analysis. CW and BA participated in the design and coordination. All authors read and approved the final manuscript.

\section{REFERENCES}

1. Blaum WE, Jarczweski A, Balzer F, Stotzner P, Ahlers O. Towards Web 3.0: taxonomies and ontologies for medical education - a systematic review. GMS Z Med Ausbild 2013;30(1):Doc13.

2. Cook DA, Erwin PJ, Triola MM. Computerized virtual patients in health professions education: a systematic review and meta-analysis. Acad Med 2010 Oct;85(10):1589-1602.

3. Hempel G, Neef M, Rotzoll D, Heinke W. Study of medicine 2.0 due to Web 2.0?! - Risks and opportunities for the curriculum in Leipzig. GMS Z Med Ausbild 2013;30(1):Doc11.

4. Sucha M, Engelhardt S, Sarikas A. Internet discussion forums as part of a student-centred teaching concept of pharmacology. GMS Z Med Ausbild 2013;30(1):Doc2.

5. Hillenburg KL, Cederberg RA, Gray SA, Hurst CL, Johnson GK, Potter BJ. E-learning and the future of dental education: opinions of administrators and information technology specialists. Eur J Dent Educ 2006 Aug;10(3):169-177.

6. Handal B, Groenlund C, Gerzina T. Dentistry students' perceptions of learning management systems. Eur J Dent Educ 2010 Feb;14(1):50-54.

7. Cowpe J, Plasschaert A, Harzer W, Vinkka-Puhakka H, Walmsley AD. Profile and competences for the graduating European dentist-update 2009. Eur J DentEduc 2010 Nov;14(4): 193-202.

8. Sanz M, Widstrom E, Eaton KA. Is there a need for a common framework of dental specialties in Europe? Eur J Dent Educ 2008 Aug;12(3):138-143.

9. Plasschaert AJ, Lindh C, McLoughlin J, Manogue M, Murtomaa H, Nattestad A, Sanz M. Curriculum structure and the European Credit Transfer System for European dental schools: part I. Eur J Dent Educ 2006 Aug;10(3):123-130.

10. Plasschaert AJ, Manogue M, Lindh C, McLoughlin J, Murtomaa H, Nattestad A, Sanz M. Curriculum content, structure and ECTS for European dental schools. Part II: methods of learning and teaching, assessment procedures and performance criteria. Eur J Dent Educ 2007 Aug;11(3): 125-136.

11. Mattheos N, Nattestad A, Falk-Nilsson E, Attstrom R. The interactive examination: assessing students' self-assessment ability. Med Educ 2004 Apr;38(4):378-389.

12. Leisnert $\mathrm{L}$, Mattheos $\mathrm{N}$. The interactive examination in a comprehensive oral care clinic: a three-year follow up of students' self-assessment ability. Med Teach 2006 Sep;28(6):544-548.

13. Davidson LK. A 3-year experience implementing blended TBL: active instructional methods can shift student attitudes to learning. Med Teach 2011;33(9):750-753.

14. Brich J. Akzeptanz und Auswirkungen von Team-based Learning im Fach Neurologie: eine Pilotstudie. GMS Z Med Ausbild 2013;30(2):Doc20.

15. Eberhard L, Hassel A, Baumer A, Becker F, Beck-Mubotter J, Bomicke W, Corcodel N, Cosgarea R, Eiffler C, Giannakopoulos $\mathrm{NN}$, et al. Analysis of quality and feasibility of an objective structured clinical examination (OSCE) in preclinical dental education. Eur J Dent Educ 2011 Aug;15(3):172-178.

16. Brannick MT, Erol-Korkmaz HT, Prewett M. A systematic review of the reliability of objective structured clinical examination scores. Med Educ 2011 Dec;45(12):1181-1189. 